\title{
Orthogonality criterion for banishing hydrino states from standard quantum mechanics
}

\author{
Antonio S. de Castro ${ }^{\mathrm{a}, \mathrm{b}}$ \\ ${ }^{a}$ Universidade de Coimbra, Centro de Física Computacional, P-3004-516 Coimbra, Portugal \\ ${ }^{\mathrm{b}}$ UNESP, Campus de Guaratinguetá, Departamento de Física e Química, 12516-410 Guaratinguetá SP, Brazil \\ Received 16 February 2007; received in revised form 2 May 2007; accepted 3 May 2007 \\ Available online 6 May 2007 \\ Communicated by P.R. Holland
}

\begin{abstract}
Orthogonality criterion is used to show in a very simple and general way that anomalous bound-state solutions for the Coulomb potential (hydrino states) do not exist as bona fide solutions of the Schrödinger, Klein-Gordon and Dirac equations.
\end{abstract}

(c) 2007 Elsevier B.V. All rights reserved.

An alleged tightly-bound state of hydrogen with strong singularity of the eigenfunction at the origin (called a hydrino state) has received considerable attention in the literature [1]. The order of magnitude of the atomic size (Bohr radius) as well as the energy of the hydrogen atom in its ground state just derived from the Heisenberg uncertainty principle, even in a relativistic framework, should be enough to disqualify hydrino states. However, in a recent Letter, Dombey [1] rejects the solution of the three-dimensional Klein-Gordon equation, previously derived by Naudts [2], as well as the solution of the two-dimensional Dirac equation, by resorting to a few fair arguments. In addition, Dombey presents a solid argument founded on the Hermiticity of the Hamiltonian for the KleinGordon case and a suggestion of similar treatment for the threedimensional Dirac case. In the wake of Dombey's suggestion, this Letter presents such a general criterion for banishing hydrino states in the context of the standard quantum mechanics.

The time-independent Schrödinger equation

$-\frac{\hbar^{2}}{2 M} \nabla^{2} \psi+V \psi=E \psi$

and the time-independent Klein-Gordon equation

$-\hbar^{2} c^{2} \nabla^{2} \psi+M^{2} c^{4} \psi=(E-V)^{2} \psi$

E-mail address: castro@pesquisador.cnpq.br. with spherically symmetric potentials admit eigenfunctions in the form

$\psi=\frac{u_{k}(r)}{r} Y_{l m}(\theta, \phi)$,

where $k$ denotes the principal quantum number plus other possible quantum numbers, $u_{k}$ is a square-integrable function $\left(\int_{0}^{\infty} d r\left|u_{k}\right|^{2}=1\right)$ and $Y_{l}^{m}$ are the orthonormalized spherical harmonics $\left(\int d \Omega Y_{l m}^{*} Y_{\tilde{l} \tilde{m}}=\delta_{l \tilde{l}} \delta_{m \tilde{m}}\right)$, with $l=0,1,2, \ldots$ and $m=-l,-l+1, \ldots, l$, in such a way that

$H_{\text {eff }} u_{k}=\left(E_{\text {eff }}\right)_{k} u_{k}$

with

$H_{\text {eff }}=\left\{\begin{array}{c}-\frac{\hbar^{2}}{2 M} \frac{d^{2}}{d r^{2}}+V+\frac{\hbar^{2} l(l+1)}{2 M r^{2}} \\ \text { for the Schrödinger equation, } \\ -\frac{\hbar^{2}}{2 M} \frac{d^{2}}{d r^{2}}+\frac{E}{M c^{2}} V-\frac{V^{2}}{2 M c^{2}}+\frac{\hbar^{2} l(l+1)}{2 M r^{2}} \\ \quad \text { for the Klein-Gordon equation, }\end{array}\right.$

and

$E_{\text {eff }}= \begin{cases}E & \text { for the Schrödinger equation, } \\ \frac{E^{2}-M^{2} c^{4}}{2 M c^{2}} & \text { for the Klein-Gordon equation. }\end{cases}$

Meanwhile, the time-independent Dirac equation is

$H \psi_{k}=E_{k} \psi_{k}, \quad H=\vec{\alpha} \cdot \vec{p}+\beta M c^{2}+V$, 
where $\alpha_{i}$ and $\beta$ in the standard (or Dirac-Pauli) representation are given by the $2 \times 2$ block matrix form

$\alpha_{i}=\left(\begin{array}{cc}0 & \sigma_{i} \\ \sigma_{i} & 0\end{array}\right), \quad \beta=\left(\begin{array}{cc}1 & 0 \\ 0 & -1\end{array}\right), \quad i=1,2,3$,

and $\sigma_{i}$ are the $2 \times 2$ Pauli matrices. Its eigenfunction has a spinorial structure given by [3]

$\psi=\frac{1}{r}\left(\begin{array}{c}i f_{k}(r) \mathcal{Y}_{j m_{j}}^{\kappa}(\theta, \phi) \\ g_{k}(r) \mathcal{Y}_{j m_{j}}^{-\kappa}(\theta, \phi)\end{array}\right)$,

where $\kappa=\mp(j+1 / 2)$, with the minus sign for aligned spin $(j=l+1 / 2)$ and the plus sign for unaligned $\operatorname{spin}(j=l-1 / 2)$. Here, $\mathcal{Y}_{j m_{j}}^{\kappa}$ (with $j=1 / 2,3 / 2,5 / 2, \ldots$ and $m_{j}=-j,-j+$ $1, \ldots, j)$ are the orthonormalized spinor spherical harmonics resulting from the coupling of two-dimensional spinors to the eigenstates of orbital angular momentum. The normalization of the Dirac spinor $\left(\int_{0}^{\infty} d r\left(\left|f_{k}\right|^{2}+\left|g_{k}\right|^{2}\right)=1\right)$ requires that $f_{k}$ and $g_{k}$ are square-integrable functions. Using the identity $\vec{\sigma} \cdot \vec{\nabla}=\vec{\sigma} \cdot \hat{r} \frac{\partial}{\partial r}-\frac{\vec{\sigma} \cdot \hat{r} \vec{\sigma} \cdot \vec{L}}{\hbar r}$ and the properties $\vec{\sigma} \cdot \hat{r} \mathcal{Y}_{j m_{j}}^{\kappa}=-\mathcal{Y}_{j m_{j}}^{-\kappa}$ and $\vec{\sigma} \cdot \vec{L} \mathcal{Y}_{j m_{j}}^{\kappa}=-(\kappa+1) \mathcal{Y}_{j m_{j}}^{\kappa}$, there results that one can write $H_{\mathrm{eff}} \Phi_{k}=E_{k} \Phi_{k}$

where

$$
\begin{aligned}
& H_{\text {eff }}=\left(\begin{array}{cc}
\hbar c\left(\frac{d}{d r}+\frac{\kappa}{r}\right) & V-M c^{2} \\
V+M c^{2} & \hbar c\left(-\frac{d}{d r}+\frac{\kappa}{r}\right)
\end{array}\right), \\
& \Phi_{k}=\left(\begin{array}{c}
f_{k} \\
g_{k}
\end{array}\right) .
\end{aligned}
$$

It is instructive to examine the radial solutions in the neighbourhood of the origin for the Coulomb potential $-\hbar c \alpha / r$ ( $\alpha$ is the coupling constant) because $u, f$ and $g$ must behave better than $r^{-1 / 2}$ at the origin in order to guarantee their square integrability. An estimative of the asymptotic behaviour of the radial solutions for small $r$ can be obtained by neglecting the terms of order $r^{-n}$ compared with the terms of order $r^{-(n+1)}$, where $n=0,1,2$, in the Schrödinger, Klein-Gordon and Dirac differential equations.

As $r \rightarrow 0$ the terms behaving as $r^{-2}\left(r^{-1}\right)$ dominate in the Schrödinger case for $l \neq 0(l=0)$ in such a way that

$r^{2} \frac{d^{2} u}{d r^{2}}-l(l+1) u=0, \quad$ for $l \neq 0$,

$r^{2} \frac{d^{2} u}{d r^{2}}+\frac{2 M c \alpha}{\hbar} r u=0, \quad$ for $l=0$.

Substituting the Frobenius series expansion

$u=\sum_{n=0}^{\infty} a_{n} r^{\nu+n}, \quad a_{0} \neq 0$,

into (12) and (13) yields the quadratic indicial equation (obtained when $n=0): v(v-1)=l(l+1)$, which has the solutions $v_{1}=l+1$ and $v_{2}=-l$. It happens that there is no recurrence formula for $l \neq 0\left(a_{n}=0\right.$ for $\left.n \neq 0\right)$ so that the general solution for (12) can be written as $u=A_{l} r^{l+1}+B_{l} r^{-l}$. On the other hand, for $l=0$ the power series expansion fails with $v=v_{2}$ because only the first coefficient of the series can be

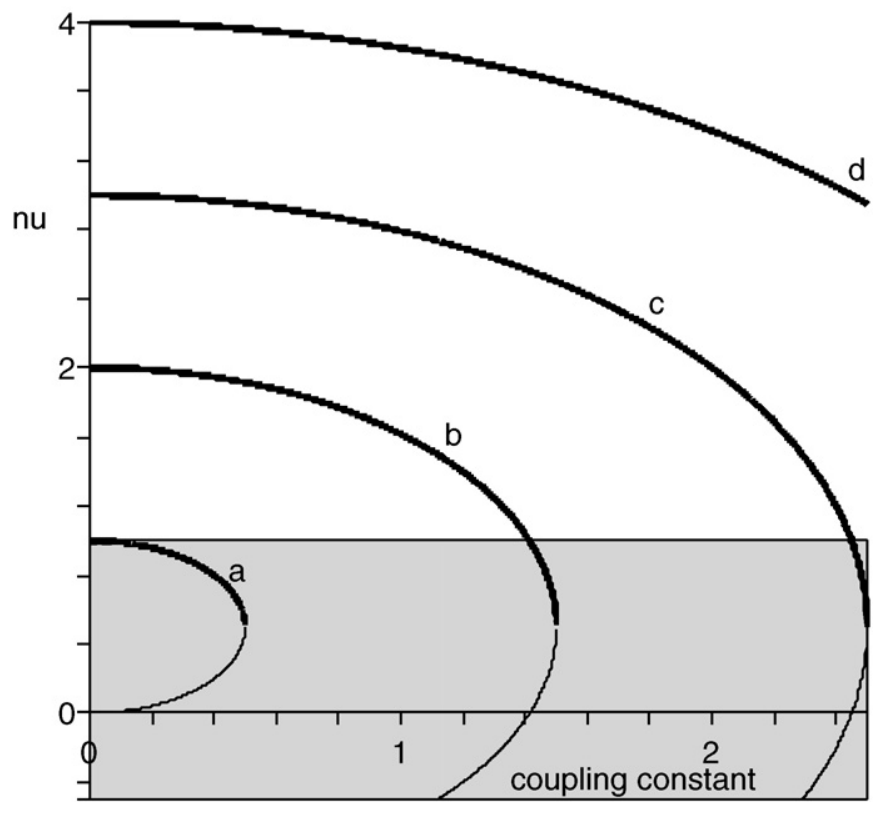

Fig. 1. The roots of the indicial equation, $v_{+}$(thick line) and $v_{-}$(thin line), as a function of the coupling constant for the Klein-Gordon case ( $a$ for $l=0$, $b$ for $l=1, c$ for $l=2$ and $d$ for $l=3$ ). The shaded area represents the zone corresponding to radial solutions diverging at the origin.

defined. It means that the Frobenius method furnishes only one solution for $l=0$. A second solution linearly independent of $u_{1}=a_{0} r+\cdots$, the solution related to $v=v_{1}$, can be found by writing $u_{2}=a u_{1} \ln (r)+\sum_{n=0}^{\infty} b_{n} r^{\nu_{2}+n}$ with $a \neq 0$ and $b_{0} \neq 0$. It follows that the general solution for $l=0$ can be written as $u=A_{0}\left(r-\frac{M c \alpha}{\hbar} r^{2}+\cdots\right)+B_{0}\left(1-\frac{2 M c \alpha}{\hbar} r \ln (r)+\cdots\right)$. The above results imply that only choosing $B_{l}=B_{0}=0$ gives a behaviour at the origin which can lead to square-integrable solutions. For short, all the normalizable radial solutions $(u / r)$ are regular at the origin and behave as $r^{l}$ as $r \rightarrow 0$, for all $l$.

As for the Klein-Gordon case, the terms behaving as $r^{-2}$, for all values of $l$, outweigh the other terms in such a manner that one can write

$r^{2} \frac{d^{2} u}{d r^{2}}-\left[l(l+1)-\alpha^{2}\right] u=0$

for small $r$. The power series expansion (14) gives the indicial equation $v(v-1)=l(l+1)-\alpha^{2}$ which has the roots

$\nu_{ \pm}=\frac{1}{2} \pm \sqrt{\left(l+\frac{1}{2}\right)^{2}-\alpha^{2}}, \quad \alpha \leqslant l+\frac{1}{2}$.

The argumentation given below is based on (16) and can be better understood by observing Fig. 1 , where $v_{+}$and $v_{-}$, as a function of $\alpha$, are plotted on the same grid. The constraint $v>-1 / 2$ which ensures the square integrability of $u$ demands that for $\alpha \leqslant 1 / 2$ there are just a S-wave $(l=0)$ solution for $v=v_{-}$although all the values of $l$ are allowed for $v=v_{+}$. Since $v<1$ for $\alpha \leqslant 1 / 2$, both S-wave solutions, in the sense of $u / r$, diverge at the origin. As $\alpha$ increases some normalizable solutions diverging at the origin for $v=v_{-}$become possible whereas some normalizable solutions for $v=v_{+}$become divergent. In any case, as the coupling constant takes critical values 


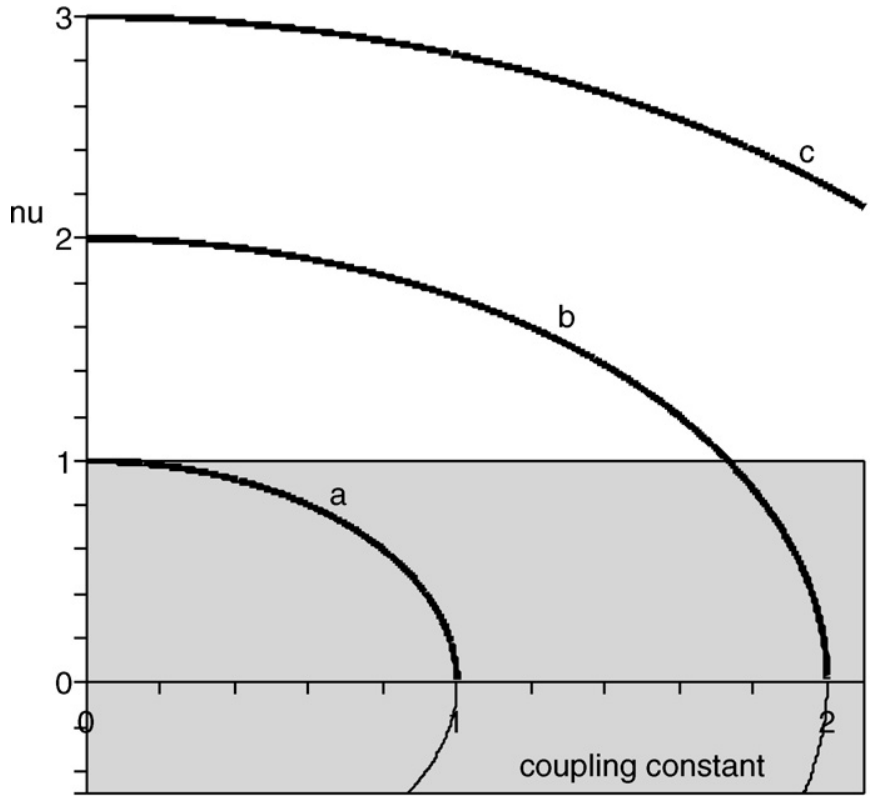

Fig. 2. The solutions of the system of indicial equations, $v_{+}$(thick line) and $v_{-}$(thin line), as a function of the coupling constant for the Dirac case ( $a$ for $|\kappa|=1, b$ for $|\kappa|=2$ and $c$ for $|\kappa|=3$ ). The shaded area represents the zone corresponding to radial solutions diverging at the origin.

$\left(\alpha=l_{c}+1 / 2, l_{c}=0,1,2, \ldots\right)$ only the solutions for $l>l_{c}$ are allowed.

In analogy with the solutions in the neighbourhood of the origin of the Schrödinger and Klein-Gordon equations, we look for solutions of the asymptotic Dirac equation

$r \frac{d f}{d r}+\kappa f-\alpha g=0$,

$r \frac{d g}{d r}-\kappa g+\alpha f=0$

in the form of power series

$f=\sum_{n=0}^{\infty} a_{n} r^{\nu+n}, \quad a_{0} \neq 0$,

$g=\sum_{n=0}^{\infty} b_{n} r^{\nu+n}, \quad b_{0} \neq 0$.

Now the coefficient of $r^{v}(n=0)$ gives the system of indicial equations

$(v+\kappa) a_{0}-\alpha b_{0}=0$,

$\alpha a_{0}+(v-\kappa) b_{0}=0$

and the two possible values of $v$ :

$\nu_{ \pm}= \pm \sqrt{\kappa^{2}-\alpha^{2}}, \quad \alpha \leqslant|\kappa|$.

Fig. 2 illustrates (20) as a function of the coupling constant for the lowest values of $|\kappa|$. The requirement of normalizability $(v>-1 / 2)$ implies that for $\alpha \leqslant \sqrt{3} / 2$ only the positive root is allowed and the radial solutions $(f / r$ and $g / r)$ are found for all the values of $|\kappa|(|\kappa|=1,2,3, \ldots)$, although the radial solutions with $|\kappa|=1$ diverge at the origin. The negative root allows divergent radial solutions with $|\kappa|=1$ for $\sqrt{3} / 2<\alpha \leqslant 1$. Nevertheless, there are no normalizable solutions with $|\kappa|=1$ for $\alpha>1$. Continuing this process, as the coupling constant increases starting with $\alpha=1$, some normalizable solutions for $v=v_{+}$turn out to be divergent whereas some normalizable solutions diverging at the origin for $v=v_{-}$become possible. As $\alpha$ takes critical values $\left(\alpha=|\kappa|_{c},|\kappa|_{c}=1,2,3, \ldots\right)$ only the solutions for $|\kappa|>|\kappa|_{c}$ are allowed. This means that the radial solutions for the smallest values of $|\kappa|$ become disallowed one after the other as $\alpha$ increases.

In the standard quantum mechanics an observable such as the energy is represented by a Hermitian operator, whose set of eigenfunctions constitutes a basis so that every arbitrary wave function can be expanded in one and only one way in terms of the eigenfunctions. Besides square-integrability, appropriate boundary conditions must be imposed on the eigenfunctions of an eigenvalue problem. Square-integrability requires that the eigenfunction vanishes at the infinity and the boundary condition at the origin for a singular potential, as the Coulomb potential, comes naturally into existence by demanding that the Hamiltonian is Hermitian, viz.

$\int_{0}^{\infty} d \tau \psi_{k}^{*}\left(H \psi_{k^{\prime}}\right)=\int_{0}^{\infty} d \tau\left(H \psi_{k}\right)^{*} \psi_{k^{\prime}}$,

where $\psi_{k}$ is an eigenfunction corresponding to an eigenvalue $E_{k}$. In passing, note that a necessary consequence of Eq. (21) is that the eigenfunctions corresponding to distinct effective eigenvalues are orthogonal. Identifying $H_{\text {eff }}$ with $H$ and $E_{\text {eff }}$ with $E$ in (21), integrating by parts and recalling the orthonormality of the spherical harmonics, it is easy to show that $u_{k}(r)$ for the Schrödinger and Klein-Gordon cases must satisfy the following constraint $[4,5]$

$\lim _{r \rightarrow 0}\left(u_{k}^{*} \frac{d u_{k^{\prime}}}{d r}-\frac{d u_{k}^{*}}{d r} u_{k^{\prime}}\right)=0$.

For the Dirac case, identifying $H_{\text {eff }}$ with $H$ in (21), one finds a constraint involving the upper and lower components, namely $[4,5]$

$\lim _{r \rightarrow 0}\left(f_{k}^{*} g_{k^{\prime}}-f_{k^{\prime}} g_{k}^{*}\right)=0$.

For the Schrödinger case, the square-integrable solution for $u\left(u \rightarrow r^{l+1}\right.$ as $\left.r \rightarrow 0\right)$ satisfies (22) and its corresponding radial solution $u / r$ is regular at the origin.

For the Klein-Gordon case, only the solutions for $u$ less singular than $r^{1 / 2}$ can satisfy the orthogonality criterion. One sees, then, that the square-integrable solution must behave as $r^{\nu_{+}}$, as $r \rightarrow 0$, where $\nu_{+}$is one of the two possibilities of (16), corresponding to the thick lines in Fig. 1. Therefore, squareintegrable solutions of the Klein-Gordon equation satisfying the orthogonality criterion are such that for $\alpha \leqslant 1 / 2$ all the values of $l$ are allowed. The $l=0$ radial solution, in the sense of $u / r$, has a divergence at the origin. As $\alpha$ increases some regular normalizable solutions become divergent. In any case, as the coupling constant takes critical values $\left(\alpha=l_{c}+1 / 2\right.$, $\left.l_{c}=0,1,2, \ldots\right)$ we are left with the solutions for $l>l_{c}$. 
For the Dirac case, only the regular solutions for $f$ and $g$ can satisfy the orthogonality criterion. This means that the square-integrable solutions must behave at the origin as $r^{v_{+}}$, where $v_{+}$is the positive square root of (20), corresponding to the thick lines in Fig. 2. Here, square-integrable solutions satisfying the orthogonality criterion are such that, for $\alpha \leqslant 1$, the radial solutions $(f / r$ and $g / r)$ are found for all the values of $|\kappa|(|\kappa|=1,2,3, \ldots)$, with the radial solutions with $|\kappa|=1$ diverging at the origin. Nevertheless, there are no normalizable solutions with $|\kappa|=1$ for $\alpha>1$. Continuing this process, as $\alpha$ increases starting with $\alpha=1$, some solutions turn out to be divergent. As $\alpha$ takes critical values $\left(\alpha=|\kappa|_{c},|\kappa|_{c}=1,2,3, \ldots\right)$ only the solutions for $|\kappa|>|\kappa|_{c}$ are allowed. This means that the radial solutions for the smallest values of $|\kappa|$ become disallowed one after the other as $\alpha$ increases.

In summary, a very simple and general criterion has been presented to reject hydrino states in the context of the standard quantum mechanics. Square integrability is sufficient enough to exclude singular wave functions in the Schrödinger equation, but not in the Klein-Gordon and Dirac ones. For the relativistic equations with the Coulomb potential, singular wave functions are allowed. The orthogonality criterion, though, imposes an additional constraint in such way that the would-be relativistic square-integrable solutions for hydrino states, related to the thin lines in Figs. 1 and 2, are not acceptable. Therefore, only radial solutions behaving at the origin as $r^{-1 / 2+\varepsilon}$, with $\varepsilon>0$, for the Klein-Gordon case, and $r^{-1+\varepsilon}$ for the Dirac case, are physically acceptable solutions.

\section{Acknowledgements}

The author is indebted to an anonymous referee for very constructive remarks and to CNPq and FAPESP for partial financial support.

\section{References}

[1] N. Dombey, Phys. Lett. A 360 (2006) 62, and references therein. [2] J. Naudts, physics/0507193.

[3] W. Greiner, Relativistic Quantum Mechanics, Wave Equations, Springer, Berlin, 1990.

[4] K.M. Case, Phys. Rev. 80 (1950) 797.

[5] D. Xianxi, et al., Phys. Rev. A 55 (1997) 2617, and references therein. 